\title{
IoT-Based Smart Health Unit
}

\author{
Syed Muhammad Waqas Shah, Maruf Pasha* \\ Department of information technology, Bahauddin Zakariya University, Multan, Punjab, Pakistan. \\ * Corresponding author. Tel.: +923467111731; email: maruf.pasha@bzu.edu.pk \\ Manuscript submitted July 10, 2016; accepted October 12, 2016. \\ doi: $10.17706 /$ jsw.12.1.45-32
}

\begin{abstract}
The World Wide Web has made world a global village and internet of things (IoT) is making this place smarter and efficient by allowing the variety of sensors and smart objects to collect and process the information for various use. These smart objects are making healthcare system smarter and giving advantages to underdeveloped countries where basic health units do not have complete medical infrastructure. However, a specialized architecture is missing for smart health unit that can support for collecting and forwarding the health information to headquarter hospitals where live support for patient is available. In this paper, we evaluated the various IoT based health system models and their applications. To provide intelligent health services, we have proposed a smart health unit model in which the country's basic health units are converted into smart health units and these smart health units are connected to their respective headquarter hospitals. The proposed model presents a layered approach, which includes sensor, network access and service layer that make the efficient communication possible.
\end{abstract}

Key words: BAN, cloud services, healthcare, IoT, remote monitoring, smart health unit, WBAN.

\section{Introduction}

Internet of Things(IoT) made this possible to connect various smart objects to send and receive information [1]. We see an extensive use of these smart objects like smart phones, tablets, sensors and many other wired and wireless types of equipment. Theses smart objects are used for various purposes, for example a smart phone can be used for accessing weather information monitored by the sensors, can monitor room or body temperature, can make an audio and video call and can retrieve and store the data over cloud storage thus the use of these smart objects is not limited.

The use of IoT technology has been extended from body to smart home, smart city and smart environment. These smart environments include sensors for infant monitoring which provides real time information about body temperature, body activity level and body position of the infant on the parent's smart phone. Smart home includes home security monitoring remotely or locally on smart phone and various sensor networks are deployed to monitor weather, earth quick, tsunami etc. Smart phone ranges of sensors include accelerometer, gyro, video, proximity compass and GPS. RFID tags can also be used to identify and authenticate the resources in health care system [2]. Many countries and health industries started some IoT based projects for example; China Europe, Japan, UK and Italy. IoT technology can be used to provide the basic health services at minimum cost.

IoT technology in health care system cast a great impact on underdeveloped countries where basic health services are not available at door steps especially in rural areas. Internet facility is available almost everywhere in the world and underdeveloped countries also contain public switched telephone network and mobile operators with wired or wireless infrastructure, this facility can be utilized to provide basic smart health services in rural areas.

Patients in smart health unit can be treated remotely when physicians or specialists are not available. Physician at remote site can interact with patient in smart health unit via internet using some state of the art 
applications and physician can get real time data of patient collected by sensors at smart health unit and can suggest medicine or some further investigations. This will also help the poor patients not to go far distance hospitals for normal or routine checkup. Patient will only travel if physician recommends. This implementation model will provide the health facility at doorstep to the public with great economical impact on both government investment and public expenditures and collectively on country's economy. This smart health unit solution will not only provide the basic health facility to the patient but will also facilitate the physician to take auto decisions. Machine learning techniques will be used to take auto decisions on the cloud server by using cloud services. Cloud services will also provide the remote storage and machine learning techniques to maintain the patient's database and auto decisions. Another benefit for the government is to keep track of the entire nation's health.

Main dream of this paper is to use the PSTN and mobile operator's infrastructure with IoT technology to provide basic health services at door step especially in under developed countries and rural areas. In this paper, we proposed an implementation model for IoT-Based smart health care units named as smart health unit (SHU).This model is implementable where basic health units are available by joining the sensors in basic health units to the tehsil headquarter hospitals (THQ) and THQ to the district headquarter (DHQ) which are administrative division of areas by using internet that is provided by PSTN or mobile operators. Model is presented in Fig. 1. Further work is divided in following sections: Section 2 includes the associated work; Section 3 presents our suggested three layers IoT-based SHU model; Section 4 case study; and conclusion in Section 5.

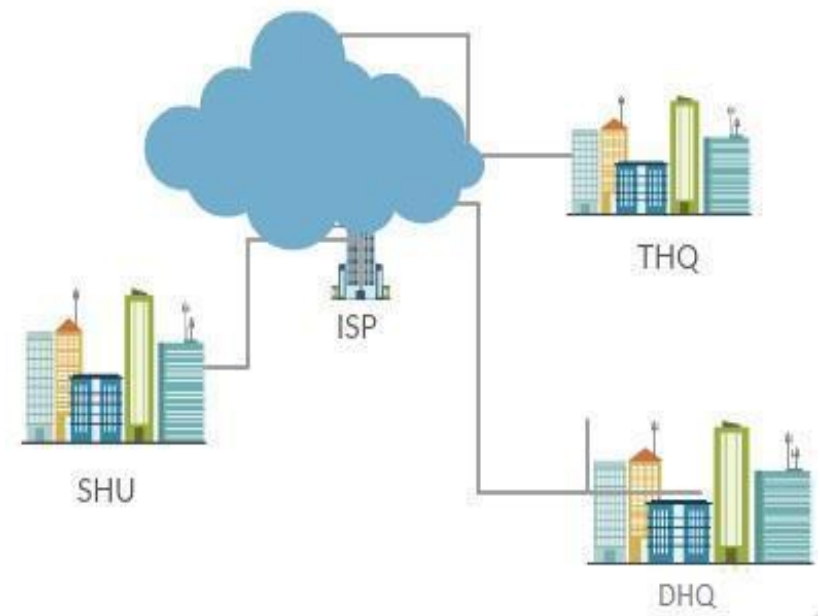

Fig. 1. Smart health unit model.

\section{Associated Work}

As highlighted in previous section IoT technology, with the use of sensors provides an important advantage in the field of medical. Many related work has been done already which is analyzed on the basis of their application domain, security and efficiency in this section.

\subsection{Application Domain}

Remote monitoring mechanism for elderly people is developed by Wei et al [3]. Mechanism provides the way of remote and continuous monitoring of elderly patients. Sensor devices collect the health information required for monitoring of blood pressure from the body of a patient. Collected information is processed to the storage system. Storage system is also equipped with state of the art applications. So, storage system also generates intelligent warning messages to the relevant bodies when health condition crosses a specified level. Machine learning techniques are also used to predict the health condition of patient. This alerts the patients and doctors in advance to take precautionary measurements. So, it is helpful for patients suffering from high blood pressure diseases, as they critically need to know their health conditions. 
Ganchev et al [4] provided a framework for deploying smart city projects. Core concept of providing smart services includes an IoT service provider. An IoT service provider provides all services to the smart city. These services are grid station monitoring, road traffic monitoring, security monitoring, commerce services, tourism management and smart education. The centralized IoT service provider also manages health services in smart city. IoT service provider is connected to databases of all hospitals in city. These databases contains all the information about health services in a hospital for example, available medical staff, physicians, pharmacists, specialists, ambulance service, available bed and room for patient and hospital's total capacity to handle various type of patients.

Smart health services help in monitoring the patient remotely some application features also increases the smartness and efficiency of IoT based smart health system. An efficient rehabilitation system proposed by Fan et $a l[5]$ is also an optimized system. Their proposed system manages resources by providing semantic information. Ontology based system make quick and efficient integration of resources possible. Various smart and wireless devices communicate in the smart rehabilitation system by using TCP/IP protocol suite. System can be managed in three level of hierarchy. The included levels are Management level, optimization level and computer-human interaction level that increases the efficiency of smart health system.

Another, help to you (H2U) health care system is presented by Basanta et al. [6]. Authors provide the basic framework for monitoring of elderly people and patients. Usually doctors cannot monitor the patient all the time so, there is need to monitor elderly people especially elderly patients at their home. Help to you health care system connects heterogeneous type of computing devices together. As elderly patients need continuous monitoring, a wide range of sensors can be connected to the body of patient. Mobile apps are used to store the health information collected through sensors. This information can be submitted to the central database continuously or only when need it. Stored information is accessible from everywhere by using Internet. Auto decision facility is also provided which helps in generating emergency alarms. Alarms messages are forwarded to the relevant persons like relatives or doctors. Sensors collect body temperature, body weight, blood pressure, heartbeat and body sugar. This information is used by the medical professionals to take relevant decisions.

Tyagi et al. proposed a conceptual model for Cloud and IoT based smart healthcare system [7]. Conceptual work was consisted on variety of healthcare applications. For example, electronic health record to generate and store the health record automatically, personal health record to record the health states of patient, clinical decision system to automate the decision process on the basis of patient's record and pharmacy system to prescribe the medicine on the basis of diagnostic symptoms.

Amendola et al. [8] presented a survey work for RFIDs applications in health care system. Environmental changes like temperature and humidity can also be detected and monitor with specialized RFIDs. Environmental pollution can be detected as some specialized RFIDs can monitor the gases. As pollution causes harm to the health of people so detection of pollution indirectly monitors health of people. These RFIDs can also be used in smart health care system for diagnostic purposes as specialized RFIDs sense the gases so they can also be used to differentiate between healthy and polluted breath of human being and also other living organisms.

Another model to provide remote monitoring health services is developed by Swiatek and Rucinski [9]. Model mainly focuses on delivering smart health services by using distributed environments. Service model delivers smart health services with innovative and commerce aspects of e-health services. They tried to maintain the delivery of complex services in distributed environment. Health services are delivered by collecting vital health information on the smart mobile device. Smart phones are used to collect health information from the body of patient. Health information can be collected by using smart phones which have smart sensors to sense the body.

\subsection{Efficiency}

As continuously data processing is required to provide non stop smart health services. Hardware must be capable to support various communication protocols. To support communication between different entities it is possible either to deploy new protocols supportable by different hardware entities or to develop new hardware to support new protocols. Supporting existing hardware is more better way as it is a cost effect solution than 
developing a new hardware. Jara et al [10] presented a new integrated framework with a fine tuned communication protocol to provide efficient non-stop smart health services. A specialized hardware gateway is implemented to connect the internal local network to the outside wide area network. This specialized gateway fulfills the requirement of efficiency. Gateway supports various technologies in local network and wide area network so it works as a two-way connection entity. Connection between body sensors network and this specialized gateway can be established through various technologies like ZigBee or Bluetooth and from gateway to internet it supports TCP/IP and many other protocols and technologies.

Yang et al. [11] Proposed a service model to provide efficient IoT based health services. Model is divided into three service layers to collect the data from patient's body, to manage the resources of health system and to provide the medical services in response to the collected data by using iMedBox, iMedPack and Bio-Patch. Model interacts with different aspects of health information systems like hospitals, emergency management and Patient's body. Bio-Patches hold the modern sensors that are used to collect health information from body of a patient. Patient registration takes place at iMedBox and registered patient can get relevant remote medical prescription through iMedBox obtained from database.

Kaur et al. presented a new framework for the development of smart cities by integrating cloud-computing and IoT technologies [12].They used different type of cloud services in their presented model for infrastructure, network, storage and also application software from cloud. But they did not address the security concerns related to smart cities.

$\mathrm{Xu}$ et al. proposed a semantically rich model to access the data in IoT-based emergency health management system [13]. They used XML language to provide the semantic information about the data. Resources are managed efficiently as they are defined explicitly. A person can access the information about any physician, ambulance service and hospital. Medical staff can also serve remotely. They also proposed a layer model for efficient functionality of each data accessing activity at cloud. Layer model provide the mechanism to handle the data of different patient and doctors at different hospitals at cloud. As there are different data sources and resources so differentiation between data is also managed in this model.

\subsection{Security}

Location and electronic health record of a patient should be kept confidential. As this information can be used for various harmful purposes. For example, a thief, terrorist or a hacker can use it for his own purposes. A security framework for maintaining confidentiality of electronic health record and location is presented by Ding et al. [14]. They discussed the various security concerns to the smart health environments. But they mainly focused on the security of location, personal identity, query identity and electronic health record of a patient. For queries they suggested the use of some privacy tools and protocols to successfully accomplish the query in a secure way. They also suggest some statistical techniques to infer the security level and behavior of the participants of health environment.

Gong et al. presented a privacy protected smart healthcare model by implementing a lightweight private homomorphism and improved encryption DES algorithms [15]. These algorithms only proved the confidentiality to the data not authentication. Proposed algorithms only applicable when there is need to access the data from server to the inquirer.

Gope et al. proposed a secured healthcare system based on internet of things [16]. Their model provides data integrity, authentication, confidentiality and secure localization. They used offset code book (OCB) to provide security and integrity to the data. Lightweight Anonymous Authentication Protocol (LAAP) to provide authentication, anonymity and secure localization with reduced computational overhead.

Chiuchisan et al. proposed a specialized framework for the monitoring of patient at intensive care unit [17].They made use of wire sensors to collect the data from body of patient. Collected information is sent to the monitor node which displays the condition of the body. In emergency condition, alarms can be generated over the smart devices of medical professionals. Use of wired sensors is secured but can be misplaced from the body of patient. 
Doukas et al. addressed and proposed an efficient mechanism for establishing the security in IoT-based smart health system [18]. To lower the computational and memory overhead at sensor devices they shifted the encryption overhead towards gateway device. This full fill the requirement of computing power and memory for the encryption process. So, collected information will always be encrypted at this device. PKI is used to encrypt the data and digital certificates for authentication purposes.

Table 1. Associated Work Analysis on Given Metrics

\begin{tabular}{|c|c|c|c|}
\hline References & Application & Security & Efficiency \\
\hline [3] & $\begin{array}{l}\text { Remote Monitoring } \\
\text { Emergency alert. }\end{array}$ & No & Yes \\
\hline$[4]$ & $\begin{array}{l}\text { Remote monitoring } \\
\text { Emergency alert. }\end{array}$ & No & Yes \\
\hline [5] & $\begin{array}{l}\text { Remote monitoring. } \\
\text { Remote monitoring }\end{array}$ & Yes & No \\
\hline [6] & $\begin{array}{l}\text { Emergency alert, } \\
\text { Medication reminder. } \\
\text { Remote monitoring, }\end{array}$ & No & No \\
\hline [7] & $\begin{array}{l}\text { Auto health recording, } \\
\text { Auto prescription. }\end{array}$ & No & Yes \\
\hline [8] & $\begin{array}{l}\text { Remote monitoring, } \\
\text { Body pattern recognition. }\end{array}$ & No & No \\
\hline [9] & $\begin{array}{l}\text { Remote monitoring, } \\
\text { Commerce aspect. }\end{array}$ & No & No \\
\hline$[10]$ & Remote monitoring. & No & Yes \\
\hline [11] & $\begin{array}{l}\text { Remote monitoring, } \\
\text { e-prescription, } \\
\text { Medication reminder. }\end{array}$ & No & Yes \\
\hline [12] & Remote monitoring. & No & Yes \\
\hline [13] & $\begin{array}{l}\text { Remote monitoring, } \\
\text { Emergency management. }\end{array}$ & No & Yes \\
\hline$[14]$ & Remote monitoring. & Yes & Yes \\
\hline [15] & Remote monitoring. & Yes & No \\
\hline [16] & Remote monitoring. & Yes & No \\
\hline [17] & $\begin{array}{l}\text { Remote monitoring, } \\
\text { Emergency management. }\end{array}$ & Yes & Yes \\
\hline [18] & Remote monitoring. & Yes & Yes \\
\hline
\end{tabular}

\section{Proposed Model}

The smart health unit model comprises of three layers for collecting, storing and accessing the patient's health data. Participants in each layer of smart health unit are also presented in table 1 with their functions and respective protocols.

\subsection{Data Collection}

Sensor Layer: The basic interface layer that interacts with the patient at smart health unit is called a sensor layer. This layer interacts with patient and upper layers. Body sensors in this layer of proposed model collect health information like heart beat, respiratory rate, glucose level, blood pressure and much more. Service access layer will be respondent on the basis of this sensed information to take relevant decisions by the medical staff. Many sensor devices may work on this layer. Sensors in this layer are used to sense the information from patient's body and also from surroundings of the patient's body in smart health unit. Sensor layer interacts with network access layer through variety of protocols depending upon the requirement. The communication protocols at this layer are ZigBee, Bluetooth but variety of protocols and standards can be used. For example IEEE wireless standards for local area communication. 


\subsection{Data over Cloud}

Network Access Layer: Core Network is accessed using this layer. This layer connects the devices in sensor layer with service provider by making use of different devices, standards and protocols that may include TCP/IP, Dslam, Dsl and 3G/4G connections. In smart health unit data is collected from patient's body will be transmitted to the cloud storage using mentioned protocols. Many Internet service providers also providing cloud services. This may be data storage and query services to query the data in cloud storage. The gateway device in the smart health unit sends the data to a specified cloud server over the Internet. From cloud storage data will be accessed by remote Tehsil Head Quarter Hospital, District Head Quarter Hospital or anywhere from the earth. Even in the absence of doctor or medical professionals in tehsil or district headquarter hospital patient will be able to communicate with medical professionals on smart mobile devices. We can also make use of virtual private network over public network to emulate the functionality of being connected together in a single network. VPN may also connect DHQ, THQ hospitals and smart health unit for providing live support to the smart health unit.

\subsection{Data Access}

Services Access Layer: Patient data can be accessed from THQ, DHQ hospitals or from anywhere using different application protocols. Patient's stored information will be queried using this layer by the doctors or patient himself. This layer will provide the opportunity to directly communicate with patient on the smart health unit by using different audio/video applications like Skype or health sessions via video conferencing. As cloud storage will store the patient's health information so this information can be accessed on smart devices in THQ/DHQ by using query applications provided by the cloud service providers. Cloud services may also include auto decision actions and alarm message generation if patient is being monitor continuously at remote end.

Table 1. Proposed 3-layer Model with Function, Participants and Protocols

\begin{tabular}{llll}
\hline \hline Layer & Participants & Function & Protocols \\
\hline \multirow{2}{*}{ Sensor Layer } & $\begin{array}{l}\text { Sensors, } \\
\text { RFIDs. }\end{array}$ & Collects the data from body. & ZigBee, Bluetooth etc. \\
Network Access Layer & $\begin{array}{l}\text { Gateways, } \\
\text { Cloud Servers. } \\
\text { Doctors, Patients, } \\
\text { Service Access Layer }\end{array}$ & WAN access, Access to cloud. & TCP/IP, 3G, 4G, \\
& Admintrators. & Service access interface. & HTTP, HTTPS. \\
\hline \hline
\end{tabular}

\section{An Implementation Scenario in Pakistan}

Pakistan is an under developed country with an estimated population of 191 million. More than $60 \%$ of population resides in rural areas [19] with almost $90 \%$ of Internet facility through mobile operators and public switched telephone network but complete medical facility is not available at doorstep. For medical facilities, rural areas are divided in basic health units at union council level, tehsil headquarter hospital at tehsil level and districts headquarter hospital at district level for administrative purposes. But complete medical staff and equipment is not available at every basic health unit. So, patient has to travel to urban areas even for basic health checkup.

In given scenario, a patient in rural area visits to basic health unit which is converted to smart health unit (SHU) .At smart health unit body sensors will be attached to the body of patient for collecting information that is compulsory for health checkup like body temperature, pulse rate and heart beat etc. Collected information is sent to the cloud storage over the internet by using mobile operators network or public switched telephone network. The doctor will access this information or medical staff at tehsil headquarters or district headquarters hospitals, which is shown in figure.3. Doctor will analyze the collected information and will give response to the patient with some prescription of medicine or suggestion for further investigations. Furthermore, doctor at THQ/DHQ hospital communicates and interacts with the patient at SHU through video call. This live interaction with patient is too much beneficial in understanding of patient's history. 


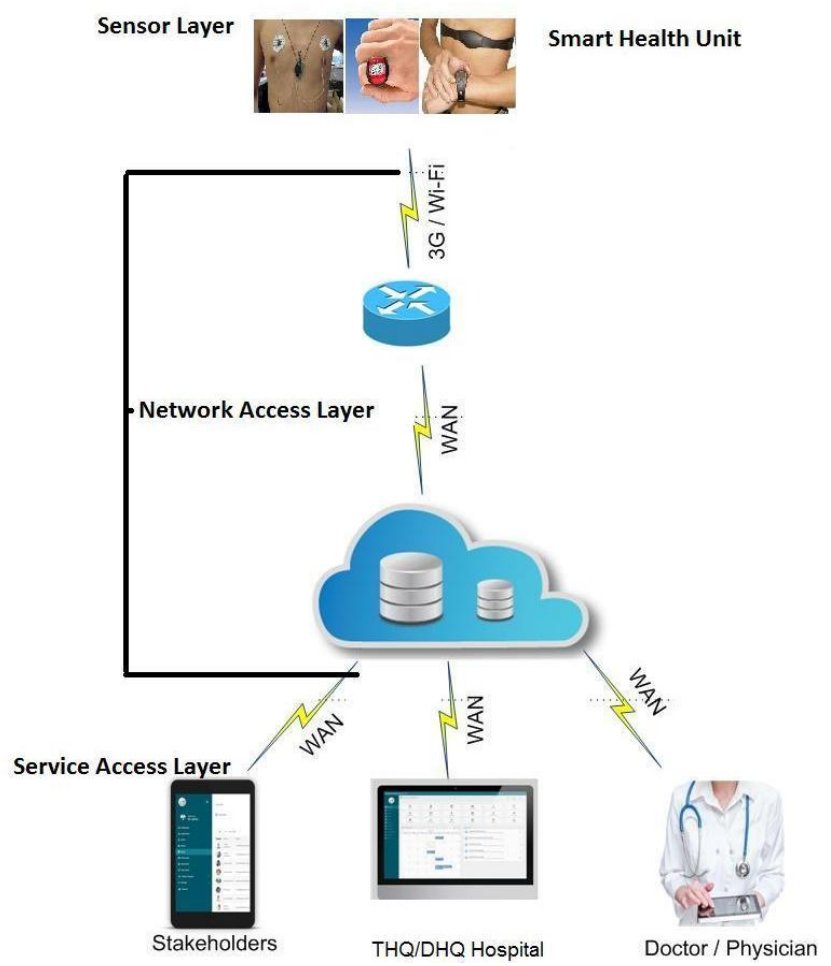

Fig. 3. Implementation scenario of smart health unit.

\section{Conclusion}

IoT based smart health units projects are widely in discussions. Implementation of e-Health and m-Health on large scale in the world has proved the acceptance of IoT-based health services. Various architectures for e-Health and m-Health are proposed with pros and cons. Our proposed model is specialized for providing basic health services and access to the medical staff residing at far distance from patient's area. Patient can visit to the smart health unit and interacts with doctor through audio and video applications like Skype. We have proposed a three-layer model; sensor layer, network access layer and service access layer to divide the communication functionality for better understanding and implementation.

Our future work will include implementation of this proposed model with data security. We are also investigating more issues to provide IoT-based smart health services where internet facility is not available in rural areas.

\section{References}

[1] Kortuem, G., Kawsar, F., Fitton, D., \& Sundramoorthy, V. (2010). Smart objects as building blocks for the Internet of things. IEEE Internet Computing, 14, 44-51.

[2] Mok, E., Guenther, R., \& Chen, W. (2012). Initial test on the use of GPS and sensor data of modern Smartphone for vehicle tracking in dense high rise environment. IEEE Transactions on Ubiquitous Positioning, Indoor Navigation, and Location Based Services.

[3] Wei, Z., Chaowei, W., \& Nakahira, Y. (2011). Medical application on internet of things. Proceedings of the IET International Conference on Communication Technology and Application (pp. 660-665).

[4] Ganchev, I., Ji, Z., \& O'Droma, M. (2013). A generic IoT architecture for smart cities. Proceedings of the Irish Signals \& Systems Conference 2014 and 2014 China-Ireland International Conference on Information and Communications Technologies ( pp.196-199).

[5] Fan, Y. J., \& Yin, Y. H. et al. (2014). IoT-based smart rehabilitation system. Industrial Informatics, IEEE Transactions, 10(2), 1568-1577. 
[6] Bastana, H., Huang, Y-P., \& Lee T-T. (2016). Intuitive IoT-based H2U health care system for elderly people. Proceedings of the $13^{\text {th }}$ International Conference on Networking, Sensing and Control (pp. 1-6).

[7] Tyagi, S., Agarwal, A., \& Maheshwari, P. (2016). A conceptual framework for IoT-based care system using cloud computing. Proceedings of the $6^{\text {th }}$ International Conference on Cloud System and Big Data Engineering (pp. 503-407).

[8] Amendola, S., Lodato, R., Manzari., S., Occhiuzzi, C., \& Marrocco, G. (2014). RFID technology for IoT-based personal healthcare in smart spaces. IEEE Internet of Things Journal, 1(2), 144-152.

[9] Swiatek, P., \& Rucinski, A. (2013). IoT as a service system for e-Health. Proceedings of the IEEE 15th International Conference on e-Health Networking, Applications and Services (Healthcom), IEEE,(pp.81-84).

[10] Jara, A. J., Zamora-Izquierdo, M. A., \& Skarmeta, A. F.(2013). Interconnection framework for mHealth and remote monitoring based on the internet of things. IEEE Journal on Selected Areas in Communications, 31(9), 47-65.

[11] Yang, G. , Xie, L., Mantysalo, M., Zhou, X. , Pang, Z., Xu, L. D. et al. (2014). A Health-IoT platform based on the integration of intelligent packaging, Unobtrusive Bio-Sensor and Intelligent Medicine Box. IEEE Transactions on Industrial Informatics, 10(4), 2180-2191.

[12] Kaur, M. J., \& Maheshwari, P. (2016). Building smart cities applications using IoT and cloud-based architectures. Proceedings of the International Conference on Industrial Informatics and Computer Systems.

[13] Xu, B., Xu, L. D., Cai, H., Xie, C., Hu, J., \& Bu, F. (2014). Ubiquitous data accessing method in IoT-based information system for emergency medical services. IEEE Transactions on Industrial Informatics, 1(2), 144-152.

[14] Ding, D., Conti, \& Solanas M. A. (2016). A smart health application and its related privacy issues. 2016 Smart city security and privacy workshop.

[15] Gong, T., Huang, P., Li, Z. H. K., \& Jiang, H. (2015). A medical healthcare system for privacy protection based on IoT. Proceedings of the Seventh International Symposium on Parallel Architectures, Algorithms and Programming (pp. 217-222).

[16] Gope, P., \& Hwang T. (2016). BSN-Care: A secure IoT-based modern health care system using body sensor network. Sensors Journal, 16(5), 1368-1376.

[17] Chiuchisan, l., Costin, H-N., \& Geman. O. (2014). Adopting the internet of things technologies in health care systems. Proceedings of the 2014 International Conference and Exposition (pp. 532-535).

[18] Doukas, C., Maglogiannis, I., Koufi, V., Malamateniou, F., \& Vassilacopoulos, G. (2012). Enabling data protection through PKI encryption in IoT m-Health devices. Proceedings of the IEEE 12th International Conference on Bioinformatics and Bioengineering (pp. 25-29).

[19] Pakistan bureau of statistics, government of Pakistan. Retrieved from: http://www.pbs.gov.pk/content/area-population-density-and-urbanrural-proportion.

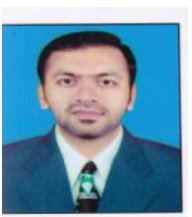

Syed Muhammad Waqas Shah received his BS in telecommunication system from Bahauddin Zakariya University, Multan, Pakistan. Mr. Waqas research interests are telecommunication and distributed systems. This research is carried out during his MS in information technology, a degree in Department of Information Technology, Bahauddin Zakariya University, Multan, Pakistan.

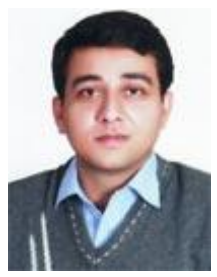

Maruf Pasha received his PhD from University of South Brittany, France. Dr. Maruf obtained his MS (IT) from NUST, Pakistan and is currently serving as head in department of Information Technology at Bahauddin Zakariya University, Multan. His research encompasses semantic web, big data and IoT systems. He has number of publications in International Journals and conferences. 